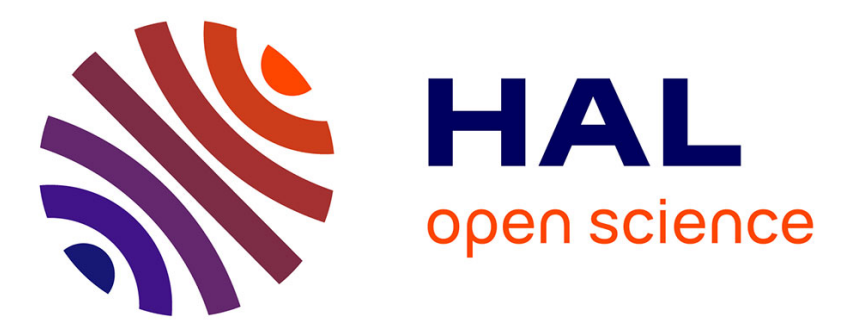

\title{
Learning from Implicit Learning Literature: Comment on Shea, Wulf, Whitacre, and Park (2001)
}

\author{
Pierre Perruchet, Stephanie Chambaron, Carole Ferrel-Chapus
}

\section{To cite this version:}

Pierre Perruchet, Stephanie Chambaron, Carole Ferrel-Chapus. Learning from Implicit Learning Literature: Comment on Shea, Wulf, Whitacre, and Park (2001). Quarterly Journal of Experimental Psychology Series a Human Experimental Psychology, 2003, 56 (5), pp.769-778. 10.1080/02724980244000657 . hal-02680330

\section{HAL Id: hal-02680330 \\ https://hal.inrae.fr/hal-02680330}

Submitted on 31 May 2020

HAL is a multi-disciplinary open access archive for the deposit and dissemination of scientific research documents, whether they are published or not. The documents may come from teaching and research institutions in France or abroad, or from public or private research centers.
L'archive ouverte pluridisciplinaire HAL, est destinée au dépôt et à la diffusion de documents scientifiques de niveau recherche, publiés ou non, émanant des établissements d'enseignement et de recherche français ou étrangers, des laboratoires publics ou privés. 


\title{
Learning from implicit learning literature: Comment on Shea, Wulf, Whitacre, and Park (2001)
}

\author{
Pierre Perruchet, Stephanie Chambaron, and Carole Ferrel-Chapus \\ University of Burgundy, Dijon, France
}

\begin{abstract}
In their analysis of complex motor skill learning, Shea, Wulf, Whitacre, and Park (2001) have overlooked one of the most robust conclusions of the experimental studies on implicit learning conducted during the last decade — namely that participants usually learn things that are different from those that the experimenter expected them to learn. We show that the available literature on implicit learning strongly suggests that the improved performance in Shea et al.'s Experiments 1 and 2 (and similar earlier experiments, e.g., Wulf \& Schmidt, 1997) was due to the exploitation of regularities in the target pattern different from those on which the postexperimental interview focused. This rules out the conclusions drawn from the failure of this interview to reveal any explicit knowledge about the task structure on the part of the participants. Similarly, because the information about the task structure provided to an instructed group of participants in Shea et al.'s Experiment 2 did not concern the regularities presumably exploited by the standard, socalled implicit, group, Shea et al.'s claim that explicit knowledge may be less effective than implicit knowledge is misleading.
\end{abstract}

Most studies on implicit learning, the process whereby people learn without intent and without being able to clearly articulate what they learn, have focused on a few prototypical laboratory paradigms (for a brief overview: Cleeremans, Destrebecqz, \& Boyer, 1998). Needless to say, beyond the investigation of these prototypical situations, the ultimate goal of the researchers is to gain knowledge about learning in a wide range of situations, including realworld settings. In keeping with this objective, several attempts have been made in the last few years to use the concepts and methods of implicit learning in order to shed light on some of the problems originating in other areas of research, such as the segmentation of language into words (Perruchet \& Vinter, 1998), the second language learning issue (Michas \& Berry, 1994), sensitivity to rhythms (Salidis, 2001) and musical structures (Tillman, Bharucha, \& Bigand, 2001), or the acquisition of orthographic regularities (Pacton, Perruchet, Fayol, \&

Requests for reprints should be sent to Pierre Perruchet, LEAD/CNRS, Esplanade Erasme, BP 26513, 21065 Dijon Cedex, France. Email: pierre.perruchet@u-bourgogne.fr

This work has been supported by the Centre National de la Recherche Scientifique (CNRS, UMR 5022), the Université de Bourgogne, and the Region de Bourgogne (AAFE).

(C) 2003 The Experimental Psychology Society 
Cleeremans, 2001). One especially promising line of research aims at exploring the relationships between the literature on implicit learning and the long-standing tradition of research concerned with motor sill learning and is represented by a set of studies performed by Wulf and coworkers (e.g., Shea, Wulf, Whitacre, \& Park, 2001; Wulf \& Schmidt, 1997).

Although there is value in this diversification of the literature on implicit learning beyond its original borders, it would appear that this generalization does not always take advantage of the methodological and conceptual advances gained from the study of prototypical situations. The studies conducted by Wulf and colleagues (e.g., Shea et al., 2001; Wulf \& Schmidt, 1997), which essentially refer to the early studies by Lewicki (e.g., Lewicki, Czyzewska, \& Hoffman, 1987), Nissen and Bullemer (1987), and Reber (e.g., 1976), provide a striking illustration of the problems that could have been avoided had the more recent literature been considered. This paper is intended to illustrate why this recent literature leads us to question some of the conclusions reached by Wulf and colleagues about implicit motor skill learning. Beyond this objective, the more general goal of the paper is to indicate the far-reaching implications of the theoretical and methodological advances in implicit learning research.

\section{The Shea et al. (2001) study}

In their recent paper, Shea et al. (2001) reported two experiments on implicit motor learning, in which participants had to move the platform of a stabilometer in order to match a changing target. In Experiment 1, in each of four successive practice sessions, the participants performed two blocks of seven 75-s trials. Unknown to the subjects, each trial was divided into three $25-\mathrm{s}$ segments. The target moved pseudo-randomly during the first and the last segments of each trial, whereas the middle segment was the same throughout the four sessions. A fifth session included a retention test, in which it appeared that Segment 2 was completed with fewer errors than Segments 1 and 3. In a subsequent interview, none of the participants mentioned that a segment had been repeated, even when they were directly questioned about this possibility. Furthermore, the participants responded randomly when they were informed about the repetition of a segment and asked to identify whether this was the first, second, or third segment. Finally, the participants were unable to select the repeated segment at abovechance level when this segment was displayed again among randomly generated segments in a subsequent forced-choice recognition test. These results essentially replicated those obtained by Pew (1974) and Wulf and Schmidt (1997) in a simpler task involving manual pursuit tracking. These studies also reported that the participants selectively improved the accuracy of their tracking on the repeated segment, although they were found to be unaware of the repeated segment and its location within a trial in subsequent recall and recognition tests.

Shea et al.'s (2001) Experiment 2 used the same task as that in Experiment 1, except that the random segment was now the middle segment, and the repeated identical segments were the initial and final ones. The authors manipulated the information given to the participants about the structure of the task. Half of the participants were informed that the first third of each trial was repeated, whereas the other half were informed that the repetition concerned the last third of each trial. In a subsequent interview, only one out of the 16 participants mentioned that another segment was repeated in addition to the one designated during the instructions. Thus this design made it possible to compare performance in instructed and noninstructed conditions, without any confound due to the position of the repeated 
segments within the sequence. It turned out that explicit instructions produced better performances in the early phase of practice, although not at a significant level. However, this pattern was reversed with practice. In the retention test in Session 5 , there were significantly fewer errors on the repeated-unknown segment than on the repeated-known segment. Thus these results show that explicit information about the structure of the task has a detrimental effect on performance.

\section{A potential drawback}

These studies provide an apparently straightforward demonstration of the possibility of unconsciously learning the structure of a complex task in a more efficient way than explicit learning permits. However, this conclusion rests on a specific condition-namely that the features of the situations that are actually responsible for the behavioural improvement have been correctly identified. To borrow Shanks and St. John's (1994) phrasing: "Before concluding that subjects are unaware of the information they have learned and which is influencing their behavior, the experimenter must be able to establish that the information he or she is looking for in awareness tests is indeed the information responsible for performance changes." The very same reasoning can be applied to the effect of explicit instructions. In order to make a comparison valid, the information given to the explicit group needs to concern the regularities that are presumably exploited in implicit conditions of learning. If the information provided by the experimenter concerns other aspects of the situation, the resulting differences in performance between so-called implicit conditions could be attributed to differences in knowledge content, rather than to the nature, implicit versus explicit, of the acquired knowledge. ${ }^{1}$

What information about the structure of the tracking tasks did Shea et al. (2001) assume to be learned in implicit conditions? An analysis of the design of the experiments, including the measures taken in the subsequent tests of explicit knowledge and the nature of the instructions given to the participants in Experiment 2, makes it clear that two features of the task were thought of as relevant: (1) the fact that the very same 25-s segment is repeated throughout the study phase (a feature whose discovery requires knowledge about the movement of the target during this segment) and (2) the location of this segment within the overall sequence (first, second, or third segment). Although the timing was changed ( 8 to $12 \mathrm{~s}$ per segment), the very same assumptions were made by Wulf and Schmidt (1997).

It should be noted that the structural features that Wulf and coworkers (e.g., Shea et al., 2001; Wulf \& Schmidt, 1997) assumed to be learned implicitly perfectly match the features they used to build the experimental situation. In doing so, Wulf and coworkers were following in the footsteps of the early investigators of implicit learning. In artificially grammar learning, Reber (e.g., 1967) posited that participants learn the finite-state grammar generating the letter strings. In sequential reaction tasks, Lewicki (e.g., Lewicki, Hill, \& Bizot, 1988) took it for

\footnotetext{
${ }^{1}$ Shea et al. (2001) never consider the possibility that the difference between instructed and noninstructed groups may be due to the fact that the two groups focus on different features of the task. Instead, they emphasize the fact that knowledge is explicit in one case and implicit in the other. For instance, they claim that "explicit knowledge may somehow be less effective than implicit knowledge", and a few paragraphs later, that "implicit information might be more effectively exploited by the perceptual motor system than explicit information" (Shea et al., pp. 858-859).
} 
granted that participants learned the rules underlying their sequences. Likewise, McGeorge and Burton (1990) assumed that participants learned that regular strings contained the digit “3”, the target feature of their material, while Kushner, Cleeremans, and Reber (1991) posited that the complex long-term dependencies that served to generate their sequence were abstracted by participants.

Our main point is the following. In all cases, subsequent research has shown that performance improvement was not based on the knowledge of the structure designed by the experimenter, but on some more or less remote by-product of the building rules. Arguably, the studies conducted by Wulf and coworkers (e.g., Shea et al., 2001; Wulf \& Schmidt, 1997) could be a fortunate exception. However, most findings in implicit learning have proven to be replicable irrespective of considerable variations in the material (e.g., consonant letters, sound pitch, spatial location of a target, etc.) and the dependent variables (e.g., ratings of preference, grammaticality judgements, reaction times, etc.) involved in the task at hand. In this context, it should be quite amazing if the deviation from a target in a pursuit tracking task followed idiosyncratic learning principles. Moreover, the structural regularities used in the Wulf and coworkers studies are similar enough to those involved in conventional situations of implicit learning to substantiate the claim that the actual content learned by participants is different from what is was assumed to be by the authors of these studies.

\section{Is it useful to know that a long segment is repeated?}

To begin with, one crucial assumption in the Wulf and coworkers studies (e.g., Shea et al., 2001; Wulf \& Schmidt, 1997) is that experimental subjects learn the whole repeated segment and notice its repetition. Indeed, in Shea et al. (2001) the postexperimental interviews are designed to assess whether subjects have detected the existence of this segment, with the recognition test contrasting the whole segment with randomly generated segments of identical length. In addition, the key information provided to the instructed subjects in Experiment 2 concerns the fact that one third of every trial is repeated throughout the session.

Recent advances in the implicit learning literature make this assumption increasingly unlikely. In artificial grammar learning, for instance, in which the material is typically composed of consonant letter strings, it has been shown that subjects do not learn the entire letter strings (which are often 6 to 9 letters long), but rather the bigrams or trigrams of which the strings consist (e.g., Perruchet, 1994; Reber \& Lewis, 1977). This aspect has also been extensively investigated in the serial reaction tasks (SRT) designed on the basis of the Nissen and Bullemer (1987) paradigm, which are of special interest to us here. Indeed, among all the situations of implicit learning, SRT studies are certainly the closest to the situation involved in the Wulf and coworkers (e.g., Shea et al., 2001; Wulf \& Schmidt, 1997) studies. In the SRT situations, a target stimulus appears in successive trials at one of a few possible positions. Participants are asked to react to the appearance of the target by pressing a key on the keyboard that spatially matches the location of the target. Unknown to participants, the same sequence of trials (typically 10) is repeated throughout the sessions. Under these conditions, experimental participants exhibit a reliable improvement in performance when compared with control participants presented with randomly generated series. As pointed out by Rosenbaum, Carlson, and Gilmore (2001, p. 459), this result closely matches that obtained in a continuous 
tracking task. ${ }^{2}$ The point is that the improved performance of participants in the SRT tasks is not due to their knowledge that the same long sequence is cyclically repeated. Instead, in these situations, participants become sensitive to (1) the frequency of individual targets, when repeated and random sequences differ on this feature (Shanks, Green, \& Kolodny, 1994) and (2) the frequency of some chunks (typically short fragments of two or three trials). Although considerable debate surrounds the question of knowing whether these chunks are available in subsequent recall and recognition tests, the idea that participants learn small chunks rather than the whole sequence remains virtually unchallenged (Buchner, Steffens, \& Rothkegel, 1998; Perruchet \& Amorim, 1992).

Applying these conclusions to continuous tracking situations is not as yet a straightforward task. Indeed, the conventional situations involve discrete elements, which provide us with natural primitives and which have no counterpart in continuous pursuit tracking. Maybe a reversal could be considered as a natural cut-off, with the segment included between two successive reversals being comparable to a letter in artificial grammar material or to a target occurrence in SRT tasks. If we accept this view, the target displacement that could be learned in this task might include one or two waveforms. Another possibility is to consider a time criterion. The approximate duration of the chunks learned in SRT tasks may be around two or three seconds (e.g., a three-trial chunk, with reaction time $=500 \mathrm{~ms}$ and Response-Stimulus Interval $=250 \mathrm{~ms}$, would last $2250 \mathrm{~ms}$ ). This value is far smaller than the 25 -s segment presented in Shea et al. (2001), or even the 10-s (or so) segment presented by Wulf and Schmidt (1997). Moreover, a comparison with the letter strings generated by artificial grammar learning shows that this value might even have been overestimated. Indeed, two or three seconds is certainly sufficient to read six- to eight-letter strings but, nevertheless, the coding units of this material have proved to be smaller. However, these direct comparisons are based on certain somewhat arbitrary choices.

A more reliable method might consist in looking for the reasons that underlie the failure of subjects to learn longer units in conventional implicit learning situations. In SRT tasks, for example, it is known that a task requiring the discovery of five-order dependency rules cannot be learned (Cleeremans, 1993). Although the interpretation of this phenomenon is not universally accepted, one generally acknowledged idea is that it involves the amount of information that can be simultaneously apprehended in one attentional focus. The size of the units would thus be directly related to the limited capacity for perceptual attentional processes (Perruchet \& Gallego, 1997). For instance, subjects would fail to encode five-order dependencies because they cannot code five successive target positions as a holistic event (if there is no salient pattern). If we accept this view, it seems difficult to believe that, in incidental training

\footnotetext{
${ }^{2}$ Making this comparison does not amount to considering that discrete key-pressing and continuous tracking tasks are equivalent from a motor control standpoint. It is likely that the two activities differ (Adams, 1987) and, moreover, it is even possible that continuous tracking involving whole-body movements differs from continuous tracking involving simpler tasks (Wulf \& Shea, in press; but see Williams \& Grbin, 1976). The key point is related, not to the perceptuo-motor component involved in coping with the task, but instead to the rule-governed aspects of the situation. In both case, the regularities are implemented as the mere repetition of a long (roughly: more than a few seconds) sequence or segment. There is, however, a minor difference because the target pattern is cyclically repeated in SRT studies, instead of being intermixed with random patterns as in the Wulf and co-workers studies. However, a few SRT studies intermixed repeated and random sequences and reported results similar to those using continuous repetitions (see Meulemans, Van Der Linden, \& Perruchet, 1998; Stadler, 1993).
} 
conditions, the length of the unit learned in continuous tracking tasks could greatly exceed a few seconds.

It should be noted that the above analysis concerns only one side of a two-sided issue. It refers to the maximum amount of data that is psychologically manageable. Another criterion is related to the internal structure of the material. In order to make learning possible, the psychologically manageable units must be large enough to be task relevant. In this context, task relevance refers to the minimum amount of data providing genuine information about the structure of the material. Generalizing the analyses performed by Perruchet and Gallego (1997) on conventional implicit learning tasks to continuous pursuit tracking, it seems that task relevance may be defined as the minimum part of the repeated segment whose frequency exceeds that of an identical part in the random segments. To make a rough estimate from a visual examination of the sample of drawings provided by Shea et al. (2001, Figures 1 or 2), any segment with a duration lower than $1 \mathrm{~s}$ is certainly not sufficient, because such a segment would apparently have as much chance of belonging to the repeated segment as to a random segment, thus making any selective improvement in performance on this segment impossible. On the other hand, it would appear to be sufficient to consider $5 \mathrm{~s}$ of the repeated segment because the chance of finding the same 5-s segment in random parts of the displacement is practically zero. The limit could be defined more precisely through a mathematical analysis of the function generating the displacement.

Further studies have to address the question of the size and the nature of the components that are learned in the pursuit tracking tasks used in the Wulf and coworkers studies. The above remarks simply provide a number of guidelines for this type of undertaking. However, all we need to note here is that all the approaches agree in concluding that these components are far smaller than the whole repeated segment. In fact, Wulf and Schmidt (1997, p. 1004) stated that it is unlikely that participants learn a motor programme covering $10 \mathrm{~s}$ of tracking behaviour, and thus the authors themselves suggest that the repeated pattern could have been parsed into smaller components. However, they do not draw the conclusion that should follow from the application of the Shanks and St. John (1994) information criterion quoted above: If performance is not based on knowledge of the overall sequence, there is no point exploring this aspect in the tests of explicit knowledge, and, likewise, giving this information to the explicit instruction group in Shea et al.'s (2001) Experiment 2 is potentially misleading.

\section{Is it useful to know the location of the repeated sequence within a trial?}

At first glance, knowing that repetition concerns, say, the second segment, would seem to be necessary if there is to be a selective improvement in performance on this segment compared to Segments 1 and 3. Such a condition would also seem to be necessary for SRT tasks. Let us assume a design analogous to that used in the Wulf et al. studies (e.g., Shea et al., 2001; Wulf \& Schmidt, 1997), in which a long sequence of target locations is divided into three subsequences, with Subsequence 2 being identical throughout the training session, and the two others randomly generated. More precisely, let us assume that, with A, B, C, and D designating the locations of the target, $\mathrm{AB}$ is regularly followed by $\mathrm{C}$ in Subsequence 2, while $\mathrm{AB}$ is randomly followed by A, C, or D in Subsequences 1 and 3 (in standard SRT tasks, immediate repetitions are not allowed). In such a design, it seems that knowing, at least approximately, 
where in the whole sequence $\mathrm{AB}$ is followed by $\mathrm{C}$ is a prerequisite for improving $\mathrm{RT}$ on $\mathrm{C}$. In fact, location knowledge is not necessarily useful, as revealed, for instance, by the reanalysis of Lewicki et al. (1988) by Perruchet, Gallego, and Savy (1990).

Let us explain the reason. Because $A B$ is regularly followed by $C$ in a subsequence that occurs iteratively throughout the training session, the chunk $A B C$ is necessarily more frequent over the entire sequence (i.e., including the repeated and the random subsequences) than are the chunks $\mathrm{ABA}$ or $\mathrm{ABD}$. As a consequence, as participants become sensitive to this feature, their responses to $\mathrm{C}$ after $\mathrm{AB}$ become increasingly shorter than their responses to $\mathrm{A}$ and $\mathrm{D}$ in the same context. This sensitivity is sufficient to allow us to observe a selective improvement of performance in the repeated subsequence. Indeed, by construction, the ABC chunks, which elicit the shortest RTs, are more frequent in the repeated subsequence than in the random subsequences. Note that this effect is obtained without the location of the repeated subsequence having been identified in any way. Paradoxically, the fact of not knowing where the fragment $\mathrm{ABC}$ occurs can even increase the differences in RT between the repeated and the random subsequences, because wrongly expecting the occurrence of $\mathrm{C}$ after $\mathrm{AB}$ during the random subsequences may have detrimental consequences on the responses to $\mathrm{A}$ and $\mathrm{D}$. It should be noted that the scenario described here can be empirically tested. It is sufficient, for instance, to compare the $\mathrm{RT}$ s on $\mathrm{C}$ after $\mathrm{AB}$ when $\mathrm{ABC}$ occurs in the repeated subsequence, on the one hand, and in the random subsequences on the other. If location is irrelevant, both measures should be identical. This was one of the tests performed by Perruchet et al. (1990) in their reappraisal of Lewicki et al. (1988), and it unambiguously demonstrated that participants did not rely on the location of regular sections in the overall sequence to improve their performance although, overall, their performance selectively improved on these sections.

To state the point in more general terms, the regular section biases the distribution of certain elementary components of the material across the whole situation, and the subject's tuning to the characteristics of this general distribution results in a selective improvement in performance on the regular section, through a purely mechanical effect. This suggests that the selective improvement in performance on the repeated section of the material in the Wulf et al. studies (e.g., Shea et al., 2001; Wulf \& Schmidt, 1997) does not mean that this section has been identified as such. Moreover, if participants expect a particular displacement-similar to that of a chunk in SRT and other tasks - whenever the context makes this possible (i.e., whenever the target is in a convenient starting position), this helps improve tracking accuracy when the expected displacement actually occurs - that is, in the repeated segment—but also impairs performance when there is a different displacement-as in the random segments. The end result is that ignorance of the location of the repeated sections, far from preventing selective performance improvement, as the authors believe, can increase the differences in performance on random and repeated segments.

\section{Conclusion}

The extension of the implicit learning issue from its original territory is obviously of value. For instance, it is clearly important to establish a link between the implicit learning literature, as characterized by the study of a few prototypical paradigms, and the literature on motor skill learning. Unfortunately, it seems that the attempt to do this in the Wulf et al. studies does not fulfil its promise. We have focused here on Shea et al. (2001), who claimed to have 
demonstrated learning without explicit knowledge of the regularities in a complex motor task (see also Pew, 1974; Wulf \& Schmidt, 1997) and, moreover, that incidental training conditions lead to better performance than when explicit information concerning the regularities is provided. We suggest that these conclusions are undermined by the fact that these studies may fail to identify what participants really learn in incidental mode. In keeping with Pew's postulates, Shea et al. and Wulf and Schmidt assumed that participants learned (1) that a long sequence was repeated from trial to trial and (2) the location of this sequence within a trial. We have shown that neither of these features is necessary for performance improvement, and, furthermore, we have pointed out that neither of these features was learned in similar previous situations of implicit learning.

Given that these features were those on which the postexperimental explicit tests focused, the conclusions of the currently available studies regarding motor learning without explicit knowledge are in need of revision. Similarly, because these features are also those about which participants in the explicit groups were informed, the evidence provided by Shea et al. about the greater efficiency of implicit learning conditions is potentially misleading. Indeed, this conclusion suggests that subjects in explicit and implicit conditions acquire the same knowledge base, with the main difference pertaining to the nature, explicit versus implicit, of this knowledge. Our reanalysis suggests a different interpretation: The difference between groups is due to the knowledge content itself. Our reappraisal suggests that the knowledge content provided to the instructed subjects is useless for performance improvement.

Arguably, the fact that explicit instructions provide irrelevant information does not directly explain why explicit instructions seemingly have a detrimental effect on performance. A comparison with the findings obtained in other implicit learning situations is again revealing. By way of an example, let us consider artificial grammar learning. It is known that instructions that lead subjects to search for rules have a detrimental effect on performance (at least when the rules are not salient, e.g., Reber, Kassin, Lewis, \& Cantor, 1980). The explanation is straightforward. The best way to improve performance in this situation consists of becoming familiar with chunks of two or three letters. This familiarity, presumably, is the natural by-product of the attentional processing of the incoming information. Now, it is probable that rule searching prevents this form of processing from occurring. Subject may, for instance, look at the first and last letters while retrieving the corresponding letters from prior items in the hope of detecting some regularities. In so doing, they never code the bigrams and trigrams, which are of primary importance for learning. The effect observed in Shea et al. (2001) is presumably similar. The instructions given to the subjects presumably engage them in irrelevant attentional processing, thus preventing implicit learning, which naturally emerges from the on-line attentional processing inherent to the requirements of the tracking task.

Of course, we are not claiming that instructions would have had positive effects, had the relevant information been provided to the subjects. Several predictions are possible, and further experimental studies are needed to explore this issue. Our point is simply that only very limited information is provided by reporting the effect of informing the subjects about aspects of the tasks that are different from those that are naturally exploited in implicit conditions. For instance, it would be quite wrong to infer from the Shea et al. (2001) study that knowledge about the task structure must be kept implicit instead of being made consciously accessible. 
Our reanalysis was prompted and supported by the literature on implicit learning that has been so abundant during the last decade or so. It is possible that the fact that this literature has focused on a few experimental paradigms may have prevented readers from understanding the general nature of the conclusions drawn from these studies. It is also possible that the endless controversies between contributors about some controversial issues, especially whether implicit learning generates conscious knowledge, have overshadowed the consensus that exists on other points. This comment represents a new opportunity to reiterate what may be the most straightforward methodological lesson gained from these studies: As a rule, subjects insist on learning things that are different from what the experimenter expected them to learn!

\section{REFERENCES}

Adams, J. A. (1987). Historical review and appraisal of research on the learning, retention, and transfer of human motor skills. Psychological Bulletin, 101, 41-74.

Buchner, A., Steffens, M., \& Rothkegel, R. (1998). On the role of fragmentary knowledge in a sequence learning task. Quarterly Fournal of Experimental Psychology, 51A, 251-281.

Cleeremans, A. (1993). Mechanisms of implicit learning: A connectionist model of sequence processing. Cambridge, MA: MIT Press.

Cleeremans, A., Destrebecqz, A., \& Boyer, M. (1998). Implicit learning: News from the front. Trends in Cognitive Sciences, 2, 406-416.

Kushner, M., Cleeremans, A., \& Reber, A. (1991). Implicit detection of event interdependencies, and a PDP model of the process. In Proceedings of the Thirteenth Annual Conference of the Cognitive Science Society. Hillsdale, NJ: Lawrence Erlbaum Associates, Inc.

Lewicki, P., Czyzewska, M., \& Hoffman, H. (1987). Unconscious acquisition of complex procedural knowledge. Journal of Experimental Psychology: Learning, Memory, and Cognition, 13, 523-530.

Lewicki, P., Hill, T., \& Bizot, E. (1988). Acquisition of procedural knowledge about a pattern of stimuli that cannot be articulated. Cognitive Psychology, 20, 24-37.

McGeorge, P., \& Burton, A. M. (1990). Semantic processing in an incidental learning task. Quarterly fournal of Experimental Psychology, 42A, 597-609.

Meulemans, T., Van Der Linden, M., \& Perruchet, P. (1998). Implicit sequence learning in children. Fournal of Experimental Child Psychology, 69, 199-211.

Michas, I. C., \& Berry, D. C. (1994). Implicit and explicit processes in a second-language learning task. European Fournal of Cognitive Psychology, 6, 357-381.

Nissen, M. J., \& Bullemer, P. (1987). Attentional requirements of learning: Evidence from performance measures. Cognitive Psychology, 19, 1-32.

Pacton, S., Perruchet, P., Fayol, M., \& Cleeremans, A. (2001). Implicit learning in real world context: The case of orthographic regularities. Fournal of Experimental Psychology: General, 130, 401-426.

Perruchet, P. (1994). Defining the knowledge units of a synthetic language: Comment on Vokey and Brooks (1992). Fournal of Experimental Psychology: Learning, Memory, and Cognition, 20, 223-228.

Perruchet, P., \& Amorim, M. A. (1992). Conscious knowledge and changes in performance in sequence learning: Evidence against dissociation. Fournal of Experimental Psychology: Learning, Memory, and Cognition, 18, 785-800.

Perruchet, P., \& Gallego, J. (1997). A subjective unit formation account of implicit learning. In D. Berry (Ed.), How implicit is implicit learning? (pp. 124-161). Oxford: Oxford University Press.

Perruchet, P., Gallego, J., \& Savy, I. (1990). A critical reappraisal of the evidence for unconscious abstraction of deterministic rules in complex experimental situations. Cognitive Psychology, 22, 493-516.

Perruchet, P., \& Vinter, A. (1998). PARSER: A model for word segmentation. Fournal of Memory and Language, 39 246-263.

Pew, R. W. (1974). Levels of analysis in motor control. Brain Research, 71, 393-400.

Reber, A. S. (1967). Implicit learning of artificial grammars. Journal of Verbal Learning and Verbal Behavior, 6, 855863. 


\section{PERRUCHET, CHAMBARON, FERREL-CHAPUS}

Reber, A. S. (1976). Implicit learning of synthetic languages: The role of instructional set. Fournal of Experimental Psychology: Human Learning and Memory, 2, 88-94.

Reber, A. S., Kassin, S. M., Lewis, S., \& Cantor, G. (1980). On the relationship between implicit and explicit modes in the learning of a complex rule structure. Fournal of Experimental Psychology: Human Learning and Memory, 6, $492-502$.

Reber, A. S., \& Lewis, S. (1977). Implicit learning: An analysis of the form and structure of a body of tacit knowledge. Cognition, 5, 331-361.

Rosenbaum, D. A., Carlson, R. A., \& Gilmore, R. O. (2001). Acquisition of intellectual and perceptual-motor skills. Annual Reviem of Psychology, 52, 453-470.

Salidis, J. (2001). Nonconscious temporal cognition: Learning rhythms implicitly. Memory and Cognition, 29, 11111119.

Shanks, D. R., Green, R. E. A., \& Kolodny, J. A. (1994). A critical examination of the evidence for unconscious (implicit) learning. In C. Umiltà \& M. Moscovitch (Eds.), Attention and performance XV: Conscious and nonconscious information processing (pp. 837-860). Cambridge, MA: MIT Press.

Shanks, D. R., \& St. John, M. F. (1994). Characteristics of dissociable human learning systems. Behavioral and Brain Sciences, 17, 367-447.

Shea, C. H., Wulf, G., Whitacre, C. A., \& Park, J.-H. (2001). Surfing the implicit wave. Quarterly Fournal of Experimental Psychology, 54A, 841-862.

Stadler, M. A. (1993). Implicit serial learning: Questions inspired by Hebb (1961). Memory and Cognition, 21, 819_ 827.

Tillman, B., Bharucha, J. J., \& Bigand, E. (2001). Implicit learning of tonality: A self-organizing approach. Psychological Reviem, 107, 885-913.

Williams, L. R., \& Grbin, I. R. (1976). The large pursuit rotor: A new gross motor tracking task. Fournal of Motor Behavior, 8, 245-250.

Wulf, G., \& Schmidt, R. A. (1997). Variability of practice and implicit motor learning. Fournal of Experimental Psychology: Learning, Memory, and Cognition, 23, 987-1006.

Wulf, G., \& Shea, C. H. (in press). Principles derived from the study of simple skills do not generalize to complex skill learning. Psychonomic Bulleting and Reviem.

Original manuscript received 21 December 2001 Accepted revision received 14 June 2002 